\title{
Importancia de la energía renovable en las generaciones futuras
}

\section{Importance of Renewable Energy for Future Generations}

Robinson Damian Angulo Jimenez², Nury Valentina García Rubiano ${ }^{3}$,
Jhon Jairo Prada Angel ${ }^{4}$, Vilmar Arturo Camacho Cortes ${ }^{5}$.

\section{Resumen}

En los sistemas de energías renovables y asequibles, o no contaminantes, se debe entender y propagar la información y educación sobre las mismas. En Colombia, un país en el que los bajos consumos de energía y emisiones generan cambios en la sociedad, ya que se pretende tener una sostenibilidad cumpliendo con los 17 oDs (Objetivos de Desarrollo Sostenibles) propuestos por la onu (Organización de la Naciones Unidas). Los hidrocarburos, el carbón, el petróleo y sus derivados tienen una importante participación en la economía y en la producción de energía para el país, por tanto, es de vital importancia comprender y propiciar educación e información en el tema, con el fin de implementar el uso de estas energías y darles un buen aprovechamiento a las mismas, cumpliendo con los oDs. Los estudios realizados asociados sobre los conocimientos que se tienen respecto a las energías renovables y el conocimiento que generamos a nivel social, demuestran un alto desconocimiento de lo que nos rodea y se puede (re)utilizar para generar energías renovables; con lo anterior, se puede sacar un estudio breve para saber en dónde se debe iniciar para

2 Estudiante, Fundación Universitaria del Área Andina, programa de Enfermería, sede Bogotá.

Correo electrónico: rangulo6/estudiantes.areandina.edu.co

3 Estudiante, Fundación Universitaria del Área Andina, Bogotá. Programa de Terapia respiratoria.

Correo electrónico: ngarcia98đestudiantes.areandina.edu.co

4 Estudiante, Fundación Universitaria del Área Andina, programa de Terapia Respiratoria, sede Bogotá.

Correo electrónico: jprada17Restudiantes.areandina.edu.co

5 Estudiante, Fundación Universitaria del Área Andina, programa de Enfermería, sede Bogotá.

Correo electrónico: vcamachođestudiantes.areandina.edu.co 
realizar un cambio cultural sobre este tema. En el 2019, Colombia inició sus acciones puntuales y contundentes comenzando con las subastas en el sector energético que le permitieron al país una apertura sin ningún precedente, marcando un antes y un después en la iniciativa del uso de energías renovables y limpias.

Palabras clave: conservación de recursos, economía verde, gestión ambiental, recursos no renovables.

\section{Abstract}

Information and education about renewable and affordable or non-polluting energy systems must be understood and disseminated. Colombia, a country where low energy consumption and low emissions produce changes in society, intends to achieve sustainability following the 17 SDGs proposed by the UN. As gas and oil, coal, and their derivatives have a significant share in the economy and the production of energy, they are highly demanded; therefore, it is vital to understand and promote education and information on the subject to implement and make good use of these energies under the SDGs. The studies associated with renewable energy and social knowledge show complete ignorance of what surrounds us and can be used as renewable energy. We can conduct a brief study to know where to start to make a cultural change. In 2019, Colombia took severe actions, starting with auctions in the energy sector that allowed its unprecedented openness, a watershed in using clean and renewable energy.

Keywords: Non-renewable resources, environmental management, green economy, resource conservation.

\section{Introducción}

El objetivo de la recopilación de la literatura es motivar a los lectores de este documento sobre el uso, la importancia y la responsabilidad que se le deben dar a los recursos y, principalmente, a las energías renovables por medio de un consumo responsable, comprendiendo los beneficios que traen en el futuro y su impacto a corto, mediano y largo plazo (Nubia-Arias, 2016).

La problemática ambiental que afecta el mundo actual es muy preocupante, debido a las diferentes posturas antropo- 
céntricas que invaden nuestra sociedad en general. Esta realidad refleja panoramas que no son los más indicados para ayudar a la supervivencia de los seres humanos, son pocas las personas las cuales piensan en estos efectos nocivos (Gerardino, Simancas y Sincelejo, 2019).

"El consumo es la acción principal la cual hacen los seres humanos en relación al uso de bienes y servicios de distinta índole, el objetivo fundamental de suplir diversas necesidades, el consumo es fundamental para formar la vida del hombre" (Nubia-Arias, 2016, p. 30). En ese sentido, posterior a la aprobación de la Agenda 2030 para el desarrollo sostenible, es necesario realizar diferentes esfuerzos con el fin de implementar la agenda de desarrollo sostenible, con la cual se puedan valorar recursos de todo tipo e implementar nuevas estrategias nacionales, regionales y globales para minimizar el consumo desaforado que existe en la actualidad (Gerardino et al., 2019).

A partir de lo anteriormente expuesto, se puede concluir que el consumidor responsable es fundamental dentro de una sociedad, a la hora de adquirir un bien servicio, puesto que se debe tener en cuenta su calidad, el impacto ambiental y conocer si el trabajo dejará una huella negativa o positiva en el ambiente es algo que el consumidor responsable debe reconocer (Pascual del Riquelme, Peñaloza y López, 2015).

La educación forma un valor fundamental a la hora de hablar de ciencias ambientales, ya que en los diferentes postulados que exponen los autores versados en el tema indican que para se debe tener en cuenta las características de la educación y población a la hora de realizar ejercicios pedagógicos; igualmente, es necesario fomentar la educación sobre el medio ambiente y el consumo responsable por parte de los docentes a tratar en las áreas respectivas (Buesaquillo, 2015).

El manejo de los residuos y del consumo responsable no debe ser una labor que se lleve solo en el contexto académico por parte de los docentes, debe ser impartido a los estudiantes con el fin de que esa información la lleven a sus hogares y allí se pueda compartir y hacer un manejo debido del consumo, el uso, la producción y el aprovechamiento de todos los productos en general (Cárdenas Sánchez, 2013).

No obstante, es necesario recalcar que para lograr el consumo responsable se necesita el conocimiento teórico y práctico, la apropiación del término debe ser visibilizada y no tergiversada, 
por lo tanto, la labor es transformar el concepto de consumo responsable y el rol docente; también, está alineado a comprender la función de la publicidad y debe ser impartida a los estudiantes (Pascual del Riquelme et al., 2015). Por eso, la tarea es visibilizar el funcionamiento del consumismo, ya que hace pasar como necesario cosas que no son de primera necesidad, con esto se vuelve una cadena de retroalimentación con el fin de que sea impartido el conocimiento a los padres (Cadena et al., 2005).

El consumo masivo de productos de desecho rápido, el desconocimiento de los términos y el consumismo por parte de los países del primer mundo y la tendencia de los países de tercer mundo a seguir los pasos de los países del primer mundo refleja una propensión a él desmejoramiento de la sostenibilidad que se pretende alcanzar para el 2030 (Herrera Araújo, 2018). Por dicha razón y ya las mencionadas, es de vital importancia generar cimientos sólidos en la educación y conciencia en los niños, estudiantes y lectores (Sabogal Aguilar y Hurtado Aguirre, 2009).

El consumo responsable de las energías y la implementación de las energías renovables y no contaminantes debe ser una solución a una de las problemáticas que se han venido evidenciando a lo largo de los últimos años, los cuales pretenden ser solucionadas con los Objetivos de Desarrollo Sostenible (Parra Cortés, 2018), por lo tanto, nos abre la perspectiva de los proyectos que son desarrollados en el presente y a futuro de dicho tema (Quijano Hurtado y Domínguez Bravo, 2008).

"La educación en energía renovable a nivel mundial es muy deficiente, y en Colombia es prácticamente inexistente, lo cual es muy preocupante" (Cárdenas Sánchez, 2013 p. 5). La propuesta de enseñanza de la energía solar como una de las fuentes de energía alternativa renovable, para estudiantes de ciclo de básica secundaria, realizada por Buesaquillo (2015) es muy pertinente en el ámbito escolar. Con esto se puede entender la importancia que tienen el uso de las energías renovables y no contaminantes, lo que implica informar y enseñar principalmente sobre el uso las mismas a las nuevas ciudadanías, a las ya existentes y a las futuras (Vilches y Gil Pérez, 2012).

Con el fin de informar a la población colombiana sobre el uso responsable de las energías no renovables, fuente importante de la contaminación (Giraldo, Vacca y Urrego, 2018), es importante concientizar a la ciudadanía que en un tiempo determinado se agotaran; de esta 
manera, es significativo promover la responsabilidad, conciencia e información del cómo poder aportar a la solución de esta problemática que nos afecta y afectará en el futuro (Rivera Forero, 2014).

El estudio realizado por Cárdenas Sánchez (2013) en un colegio en Bogotá consistió en realizar un examen con preguntas a docentes y estudiantes, de un bachillerato académico, en el cual se implementó el método experimental como modelo de investigación, concluyó que existe un deficiente conocimiento en el área de ciencias naturales sobre las energías renovables y el consumo responsable.

Para lograr un desarrollo sostenible cumpliendo con las metas de la Unesco se debe considerar que, según Quijano y Domínguez: "un elemento esencial para alcanzar el desarrollo sostenible es contar con un suministro de energía adecuado y fiable, ya que ésta es vital para erradicar la pobreza, mejorar el bienestar humano y elevar la calidad de vida de la población" (2008, p. 730). En ese sentido, se puede reflejar la gran importancia que tienen las energías renovables en cuanto a la sostenibilidad, con esto se pretende tener un planeta más sostenible, sano, reducir los consumos excesivos de productos; en general, crear un entorno saludable, fia- ble para las generaciones futuras y que se pueda realizar un desarrollo humano óptimo (Buesaquillo, 2015).

Igualmente, se deben tener conceptos claros como lo son la viabilidad de la implementación de las energías renovables en la sociedad actual y, principalmente, en la sociedad colombiana. También, entender acerca de cada una de las energías renovables (Cárdenas Sánchez, 2013), asimismo, comprender como estas son un gran aporte para dar cumplimiento a los Objetivos para el Desarrollo Sostenible de la Unesco (Quijano y Domínguez, 2008).

“Colombia ya está en el camino de la implementación de estas tecnologías y la generación a través de energías alternativas es cada vez más viable, constituyendo una de las mejores soluciones para detener el cambio climático global" (Giraldo et al., 2018, p. 11). De esta manera, es importante y es un gran avance que estas tecnologías ya se inicien a implementar en el país; sin embargo, se deben considerar diversas variantes como la economía del país, la viabilidad demográfica y territorial dentro de la implementación de las energías renovables (Quijano y Domínguez, 2008).

Es importante la concientización del tema, la educación, la información e im- 
plementación de estas nuevas tecnologías en el avance y desarrollo de los países para poder lograr la sostenibilidad y los objetivos propuestos con el fin de que sean cumplidos (Pascual del Riquelme et al., 2015).

Los Objetivos de Desarrollo Sostenible son una serie de 17 grandes propósitos sociales, económicos y medioambientales que 193 países acordaron alcanzar para mejorar las condiciones de vida en el mundo para el año 2.030. Estos fueron propuestos por Colombia, en cabeza de la entonces directora de asuntos económicos, sociales y ambientales. (Parra Cortés, 2018, p. 16)

En la actualidad, nos enfrentamos a una crisis ambiental mundial, el crecimiento acelerado del consumo mundial es una realidad de hoy; este incremento que propone la nueva ideología de consumo, "abarca a tanto a países desarrollados como a los en vía de desarrollo" (Nubia-Arias, 2016, p. 22).

La población mundial ha aumentado; este crecimiento desmedido ha provocado que los habitantes del planeta generen cambios en diversos aspectos, incluso de manera inconsciente, puesto que cada día nacen nuevas corrientes de pensamiento, nuevas inclinaciones y nuevas formas de comportamiento (Chavarro et al., 2017).
Dichos cambios se ven reflejados en diferentes sentidos; por ejemplo: el de consumo, el ser humano del siglo XXI se centra solamente en consumir sin importar qué tan caro sea un producto o si en realidad lo necesita (Nubia-Arias, 2016).

El consumo responsable, en un nivel de conciencia, busca inculcar en los comportamientos de consumo acomodándose a las necesidades reales y las del planeta, orientando a los consumidores buscar opciones que fortalezcan el medio ambiente y la igualdad social (Gerardino et al., 2019). Por esta razón, es de vital importancia tener un consumo responsable y consciente del consumo de todos los bienes y servicios adquiridos a lo largo de la vida, con el fin de generar una sostenibilidad y reducir la huella ecológica que generamos durante nuestra estadía por el planeta (Pascual del Riquelme et al., 2015).

Se debe tener presente también que las nuevas generaciones son más conscientes del impacto que genera un uso desmedido de los recursos, por lo cual, hay más compromiso en el momento de la compra de productos (Chavarro et al., 2017).

Además, se debe comprender que la huella ecológica no es solo generada por los consumidores, sino también por los fabricantes, y "esta tendencia a con- 
siderar el compromiso social y ambiental de las empresas a la hora de tomar decisiones de compra y consumo" va en aumento (Pinto Siabato, 2004, p. 3)

\section{Importancia de la educación en energías renovables}

La búsqueda de estrategias pedagógicas para la enseñanza de consumo responsable se deja a libre enseñanza según el Ministerio de Educación Nacional. No obstante, Buesaquillo considera que, "se necesita de cada una de las herramientas pedagógicas que permitan el desarrollo de estrategias no solo para disminuir el abuso y la contaminación del medio ambiente [...]" (2015, p. 14).

Resaltar y tomar conciencia de que somos responsables de nuestro alrededor, de los animales, plantas y lo que nos rodea es vital para nuestra subsistencia (Arrojo, 2006). "Los estudiantes se familiarizan con los principios básicos de las energías renovables" ( $p, 3)$ si se trata en los colegios el tema ambiental, ya que empapar a los estudiantes y crear conciencia de que ellos son los responsables del cambio climático, redunda en crear la responsabilidad sobre el consumo, ya que es un trabajo en equipo desde la escuela, la casa y su entorno (Buesaquillo, 2015).
Crear temáticas para los estudiantes en donde se incentive un consumo responsable y no desmedido es importante en la educación y para general información en todas las personas del común; según Vilches y Gil Pérez, este proceso debe darse "alejado de la publicidad agresiva que nos empuja a adquirir productos inútiles" (2012, p. 3).

Igualmente, para desarrollar las estrategias que se implementen a la hora de usar diferentes didácticas con el fin de lograr cambiar los conceptos errados que se asocian en las comunidades. En ese sentido, Buesaquillo (2015) señala la unidad didáctica sirve para cambiar las nociones "que se tengan sobre el consumo responsable no solo porque es una estrategia útil, donde se hace hincapié en la enseñanza de aprendizaje" (p. 19). Para crear la estrategia más viable es necesario formar capacitaciones especiales "como parte del compromiso que cada individuo debe tener para proteger nuestro hogar, la tierra es parte de nuestro compromiso" (Arrojo, 2006, p. 5). Por lo tanto, tener maestros en Latinoamérica y el Caribe que carecen de educación sobre el tema no es una opción, se deben tener profesores que retener los conocimientos en el área como dice Rodriguez Murcia: "Los maestros requieren conocimientos para enseñar no sólo la mate- 
ria sino también de pensar con eficacia" (2008, p. 4).

\section{Educación en los cuidados del agua de forma sostenible}

En todos los tiempos de la historia, el agua se ha sabido manejar adecuadamente, favoreciendo el desarrollo del ser humano en todas sus etapas, desde el diseño y la creación de máquinas y objetos que mejoran la función del movimiento y acción para su beneficio, incentivando el ingenio humano y su evolución (Pinto Siabato, 2004).

El agua ha sido utilizada en forma positiva para la producción de energía, ya que siempre será un recurso renovable. La evolución y las nuevas formas de extracción de energía más productiva y limpia ha generado cambios al ecosistema limitados por la industria (Arrojo, 2006).

El nuevo modelo de crecimiento económico de los países en desarrollo está basado en un consumo creciente y de explotación de recursos naturales, que generan contaminación y produciendo los nocivos gases de efecto invernadero. Estos son modelos poco eficientes y que, con comprobación científica, no concuerdan con el modelo ecológico y de sostenibilidad, pues son los causantes del cambio climático (Cadavid Rodriguez, 2015).

\section{Energías renovables en Colombia}

El uso de energías renovables es importante para el cumplimiento de los Objetivos de Desarrollo Sostenible (Gracia-Rojas, 2015), dado que el uso de energías recuperadas por hidrocarburos (el carbón) y en general las energías no renovables provocan un daño importante al medio ambiente como, por ejemplo, los gases que producen el efecto invernadero. Por esta razón, los gobiernos deben implementar el uso de energías limpias renovables (Nubia-Arias, 2016). Como señala Giraldo et al. (2018): "históricamente se originan en los combustibles fósiles: carbón, petróleo y gas natural, de ahí las consecuencias ambientales negativas por el exceso de consumo de los combustibles [...]" (p. 2).

También se debe considerar que se dé un consumo responsable de estas energías no renovables, ya que en algún punto se agotarán. Sin embargo, como dice Pascual del Riquelme et al. (2015): "considerar el compromiso social y ambiental de las empresas a la hora de tomar decisiones de compra y consumo" (p. 62). La acción de generar conciencia y sostenibilidad no depende solo de la escolarización sino, en general, de la importancia que la humanidad le dé, con el fin de que los recursos generados 
por el medio ambiente sean cuidados (García, 2018).

Se debe considerar también que según los resultados de la investigación de Quijano y Domínguez (2008), puesto que señalan que "aspectos energéticos, sociales y ambientales, cuyo resultado será la propuesta de diferentes escenarios energéticos con la valoración de la sostenibilidad global” (p. 735). Así como se mencionó es importante contemplar el buen uso, aprovechamiento y la implementación de este tipo de estrategias con el objetivo de preservar y generar una sostenibilidad del planeta (Vilches y Gil Pérez, 2012).

Giraldo et al. (2008) mencionan que, "Colombia es un país con recursos energéticos muy diversos, pero poco desarrollados y concentrados" (p. 7); con esto se puede evidenciar que Colombia es un país con los recursos necesarios para poder implementar el uso de energías renovables en el diario vivir y conseguir, como afirma Guardela y Barrios (2006), varios de los objetivos proyectados por la Unesco, reflejando la importancia de los Objetivos de Desarrollo Sostenibles para reducir la huella ecológica.

\section{Resultados}

Se recopiló información académico-científica para la realización de la encuesta, caracterizar a la población y recopilar conocimientos sobre energías asequibles y no contaminantes, que corresponde al Objetivo de Desarrollo Sostenible número siete de la ONU y preguntas de reconocimiento general; que buscan como objetivo principal comunicar acerca del conocimiento, importancia e información acerca del uso de las energías renovables. Se realizó este documento con 20 preguntas, todas ellas de carácter obligatorio y se usaron para el proceso de evaluación. Las preguntas se caracterizan en dos bloques: una de caracterización y una de conocimientos sobre oDs. La encuesta se envió a un total de 85 personas, de las cuales accedieron 40 , lo que corresponde al $47 \%$.

Para recabar los datos de la encuesta, se usó la plataforma de Google Forms con plantillas de correspondientes y criterios planteados desde el comienzo. El orden de las preguntas orientadoras fue con el objetivo de conocer la población a la cual está dirigido el proyecto de investigación y pueda ser modificando para que las personas que respondan la encuesta pertenezcan a la Fundación Universitaria del Área Andina, más exactamente a la Facultad de Ciencias de la Salud y del Deporte, seccional Bogotá. El medio de difusión de la encuesta fue por medio de redes sociales como WhatsApp, 
Facebook y Twitter. La segunda fase de preguntas enmarca los conocimientos de la oDs número siete que se refiere a energías asequibles y no contaminantes. Cabe mencionar que, se obtuvo un total de cuarenta personas encuestadas. Para la investigación realizada se generó una encuesta en la cual se realizó una caracterización de la población con preguntas claves en cuanto a nombre, edad, sexo y último grado de escolaridad alcanzado. Ya que este ejercicio investigativo también está enmarcado en el aspecto de la sostenibilidad, inmerso en el modelo de responsabilidad social de la Institución; haciendo énfasis en la población que oscila entre los 18 y 25 años, y que cursan actualmente programas de pregrado de esta universidad.

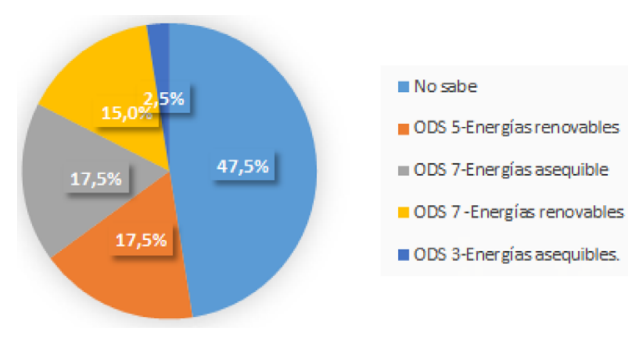

Figura 1. Pregunta 1: ¿sabe cuál de los Objetivos de Desarrollo Sostenible es el indicado para las energías renovables?

Fuente: elaboración propia.

Los encuestados respondieron de la siguiente manera a la pregunta 1 : No sabe el 47,5\%; ods 5 (energías renovables) el 17,5\%; oDs 7 (energías asequible) el $17,5 \%$; oDs 7 (energías renovables) el $15 \%$; ods 3 (energías asequibles) 2,5\%.

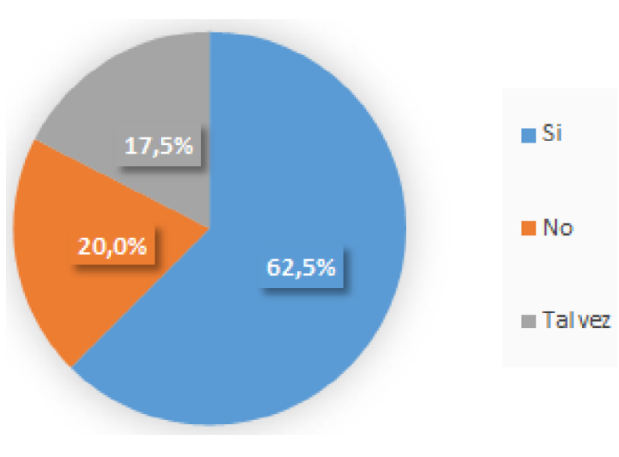

Figura 2. Pregunta 2: ¿alguna vez recibió información 0 educación acerca de las energías renovables durante su proceso de formación educativa?

Fuente: elaboración propia.

Los encuestados respondieron de la siguiente manera a la pregunta número 2 : Sí el 62,5 \%; No el $20 \%$ y Tal vez el 17,5\%.

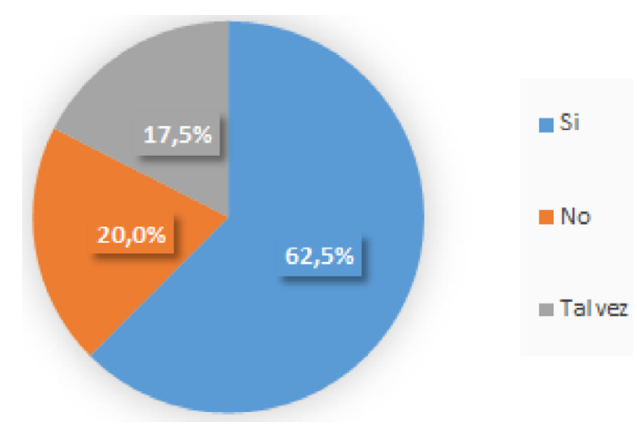

Figura 3. Pregunta 3: ¿con cuáles energías no contaminantes podemos reemplazar las usadas en el hogar?

Fuente: elaboración propia. 
Los encuestados respondieron de la siguiente manera a la tercera pregunta: Energía solar (75\%); Energía eólica $(12,5 \%)$; Gas natural (10\%) y Cocina leña o carbón $(3 \%)$.
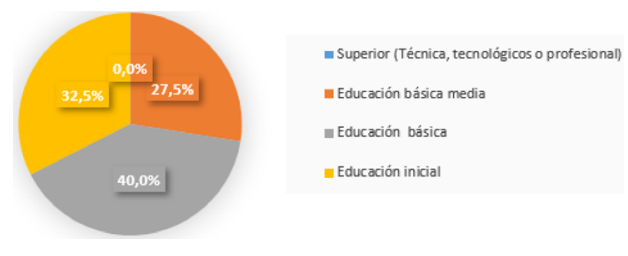

Figura 4. Pregunta 4: ¿en qué ciclo de la etapa académica está bien la implementación del concepto de “Energía renovables y no contaminantes"?

Fuente: elaboración propia.

Los encuestados respondieron de la siguiente manera la cuarta pregunta: Educación básica (40 \%); Educación inicial $(32,5 \%)$ y Educación básica media (27,5\%).

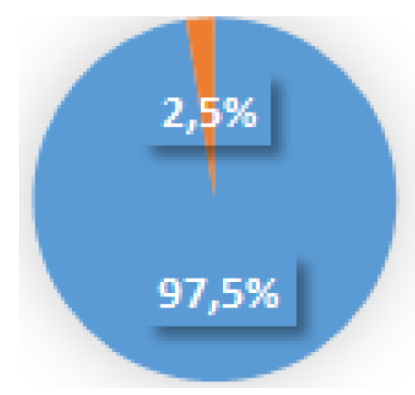

Figura 5. Pregunta 5: ¿considera importante que se enseñe el buen uso y aprovechamiento de las energías renovables y no renovables? Fuente: elaboración propia.
Los encuestados respondieron de la siguiente forma: Si el 97,5\% y No el 0,5\%.

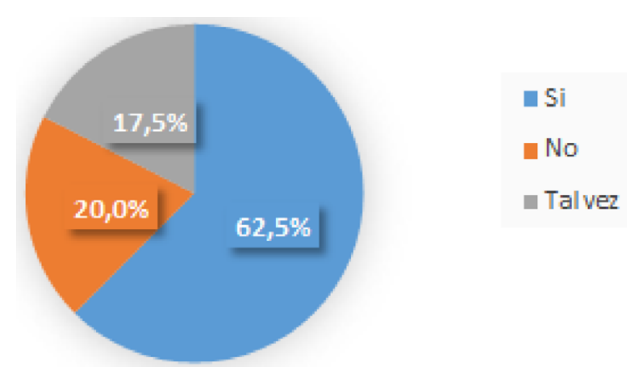

Figura 6. Pregunta 6: ¿alguna vez recibió información 0 educación acerca de las energías renovables durante su proceso de formación educativa? ¿Proceso de formación educativa? Fuente: elaboración propia.

Las respuesta a esta pregunta fueron de la siguiente manera: No el $20 \%$; Tal vez el 17,5 \% y Sí el 62,5\%.

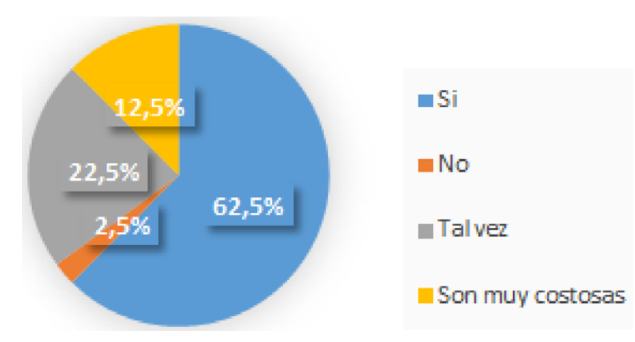

Figura 7. Pregunta 7: ¿en su hogar y en su vida cotidiana aplica 0 aplicaría el uso de energías renovables?

Fuente: elaboración propia.

Los encuestados respondieron de la siguiente forma: Sí el 62,5 \%, Tal vez el $22,5 \%$, Son muy costosas el $12,5 \%$ y No el 2,5\%. 


\section{Discusión y conclusiones}

El objeto del estudio es la inquietud sobre el desarrollo sostenible en Colombia y cómo esto puede ser implementado en a educación de los estudiantes. Los resultados indican que casi no se habla del tema dentro de un aula escolar, entonces con la recopilación de la literatura se obtuvo información sobre la enseñanza de las energías renovables en Colombia y en las diferentes edades. Se investigó de bases de datos académicas literaturas que abarcaran el tema entre el 2000 y 2019, se compararon algunos autores y sus aportes sobre la enseñanza en la escuela de los Objetivos de Desarrollo Sostenible, ya que algunos lo dejan para la vida universitaria y otros exponen que es importante el hecho de una aplicación de este tipo de conocimiento en la escuela, por medio de investigaciones, encuestas, leyendo precavidamente y recolectado los interrogantes que se pueden plantear en las escuelas (Buesaquillo, 2015).

Los resultados obtenidos en este proyecto, evidencian que anterior al haberse aplicado las dinámicas de investigación, la población de estudio no presentaba los conocimientos a plenitud sobre los oDs, llevando a implementar la necesidad de educar a la población objeto en cuanto a la importancia de las energías renovables en el futuro. Esto demuestra que la sociedad actual presenta un desorden de consumo, como parte del problema del cambio climático (Jiménez, Restrepo y Mulcue, 2019).

Por lo anterior se acepta la pregunta problema planteada: la importancia de educar a los jóvenes en cuanto a la importancia de conocer las energías renovables para el futuro.

Con base en la literatura revisada para la elaboración de este proyecto, se menciona que el crecimiento significativo mundial que se viene presentando van en aumento en la medida en que la ideología de consumo cambia, impactando en los países desarrollados como a los de en vía de desarrollo, generando una paradoja en cuanto a lo que puede causar en el medio ambiente el consumo de energía no renovables sin que contemplemos un efecto a largo plazo (Guardela y Barrios, 2006).

La misma humanidad es quien requiere una transformación urgente y a profundidad del modelo de consumo energético mundial; asimismo, sobre el consumo voraz de recursos y servicios ambientales. La educación para el consumo ambiental se fomenta como un enfoque necesario para las diferentes intervenciones en el conocimiento de la 
población, que debe estar sustentado en valores, en la responsabilidad y la concientización del consumo de los bienes de nuestro planeta (Nubia-Arias, 2016).

Por consiguiente, en la figura 5 y 6 , se ve la necesidad de reforzar la educación sobre el adecuado consumo y la importancia de las energías renovables en las poblaciones futuras. Pues, en la figura 6 , el 65,5\% respondieron que alguna vez recibieron educación sobre las energías renovables y, en la figura 5, el $97,5 \%$ respondió la importancia de la educación sobre el consumo de las energías renovables y no renovables.

Cabe mencionar que las encuestas fueron dirigidas a estudiantes cursantes del área de la salud, quienes ya presentaban un conocimiento breve, pero no a cabalidad sobre el tema.

Este trabajo nos llevó a ampliar los conocimientos sobre el tema profundizado, pues la educación es fundamental en todo el curso de nuestra vida, ya sea para conocimiento propio o de los demás, es de vital importancia la implementación en nuestro saber profesional no solo para el bien de las personas sino también de los factores que influyen en desarrollo físico y psicológico de las personas; que está ligado con la vida de nuestro planeta.
Se pudo concluir que, a pesar de que los participantes en las encuestas pertenecen a programas de educación superior en el área de la salud y tiene un conocimiento básico pro educación ambiental, queda faltando resaltar aún más la importancia y profundización sobre la realidad y la implementación a nivel mundial de las energías renovables y no renovables. También, se encontró que, como dice Ballesteros-Ballesteros (2019): "Muestra un anhelo real de fortalecer una mayor participación en el desarrollo de objetivos científicos que respondan a los problemas sociales y ambientales, pero al mismo tiempo una evaluación deprimentemente cruda de su propia impotencia para contribuir a dicha agenda" (pp. 394395). Lo cual demuestra con su trabajo realizado en las comunidades indígenas que las energías renovables deben hacer parte del cambio y la contribución a la acción de reversión del cambio climático, teniendo en cuenta que para lograr esto se debe enseñar sobre el tema y generar conciencia.

Por último, se concluye que cada día es más importante la educación ambiental y de energías renovables, puesto que la población mundial crece a ritmo acelerado sin entender las consecuencias medio ambientales que esto le acarrea a nuestro planeta. 


\section{Referencias}

Arrojo, P. (2006). Los retos éticos de la nueva cultura del agua. Polis, 14 . https://journals. openedition.org/polis/5060

Ballesteros-Ballesteros, V. A. (2019). La educación en energías renovables como alternativa de promoción del compromiso público ascendente entre los Indígenas Wayuu en la Alta Guajira. Revista Cientifica, 388-397. https://revistas.udistrital.edu.co/index.php/ revcie/article/view/14773/14712

Buesaquillo, A. (2015). Desarrollo del concepto de consumo responsable en los estudiantes del Colegio de Administración Robert Owen de los grados $8^{a}, 9^{a}, 10^{a}, 11^{a}$, ubicado en la localidad de Rafael Uribe Uribe de la ciudad de Bogotá [tesis de grado, Universidad Distrital Francisco José de Caldas]. http://repository.udistrital.edu.co/bitstream/11349/2892/1/Informe\%20Final.pdf

Cadavid Rodríguez, L. S. (2015). Aprovechamiento de los residuos orgánicos para la producción de energía renovable en una ciudad de Colombia. Energética, (46), 2328. https://revistas.unal.edu.co/index.php/ energetica/article/view/46142

Cadena, Á. I., Botero, S., Táutiva, C., Betancur, L. y Vesga, D. (2008). Regulación para incentivar las energías alternas y la generación distribuida en Colombia (Conclusiones). Revista de Ingeniería, (28), 90-98. https://www.redalyc.org/ pdf/1210/121015051012.pdf

Cárdenas Sánchez, C. C. (2013). Propuesta de enseñanza de la energía solar como fuente de energía alternativa renovable, para estudiantes de ciclo IV Básica Secunda- ria [tesis maestría Universidad Nacional de Colombia, Bogotá]. https://repositorio. unal.edu.co/handle/unal/75091

Chavarro, D. A., Vélez, I., Tovar, G., Montenegro, I., Hernández, A. y Olaya, A. (2017). Los Objetivos de Desarrollo Sostenible en Colombia y el aporte de la ciencia, la tecnología y la innovación. https://doi. org/10.13140/RG.2.2.31118.87368

Giraldo, M., Vacca Ramírez, R. y Urrego Quintanilla, A. (2018). Las energías alternativas ¿una oportunidad para Colombia? Punto de Vista, 9(13), 5. https://dialnet.unirioja. es/servlet/articulo? codigo $=6540494$

Gracia-Rojas, J. P. (2015). Desarrollo sostenible: origen, evolución y enfoques. (Documento de docencia No. 3). Bogotá: Ediciones Universidad Cooperativa de Colombia. http://dx.doi.org/10.16925/greylit.1074

Guardela Contreras, L. M. y Barrios Alvarado, L. (2006). Colombia: ¿En la vida del desarrollo sostenible? Revista de Derecho, (26), 110-136. https://www.redalyc.org/ pdf/851/85102606.pdf

Herrera Araújo, F. (2019). ODS en Colombia: los retos para 2030. Bogotá: Programa de las Naciones Unidas para el Desarrollo. https://www.undp.org/content/dam/ colombia/docs/ODS/undp_co_PUBL_julio_ODS_en_Colombia_los_retos_ para_2030_ONU.pdf

Jiménez-García, F. N., Restrepo-Franco, A. M. y Mulcue-Nieto, L. F. (2019). Estado de la investigación en energía en Colombia: una mirada desde los grupos de investigación. Revista Facultad de Ingeniería, 28(52), 9-26. https://doi.org/10.19053/01211129. v28.n52.2019.9651 
Nubia-Arias B. (2016). El consumo responsable: educar para la sostenibilidad ambiental. Aibi Revista de Investigación, Administración e Ingeniería, 4(1), 29-34. https:// doi.org/10.15649/2346030X.385

Pascual del Riquelme Martínez, M. I., Peñalosa Otero M. E. y López Célis, D. M. (2015). El consumo socialmente responsable en el mercado colombiano. Cuadernos Latinoamericanos de Administración, 11(20), 61-70. https://doi.org/10.18270/ cuaderlam.v11i20.624

Parra Cortés, R. (2018). La Agenda 2030 y sus Objetivos de Desarrollo Sostenible. Revista de Derecho Ambiental, (10), 99-121. https://doi.org/10.5354/07194633.2018.52077

Pinto Siabato, F. (2004). Energía renovable y desarrollo sostenible en zonas rurales de Colombia. El caso de la vereda Carrizal en Sutamarchán. Cuadernos de Desarrollo Rural, 1(53), 103-132. https://revistas.javeriana.edu.co/index.php/desarrolloRural/ article/view/1262

Quijano Hurtado R. y Domínguez Bravo, J. (2008). Diseño de un proyecto integrado para la planificación energética y el desarrollo regional de las energías renovables en Colombia basado en sistemas de información geográfica. En L. Hernández y J. M. Parreño (eds.), Tecnología de la Información Geográfica para el desarrollo territorial: XIII Congreso Nacional de Tecnologías (pp. 729-736). Palmas de Gran Canaria: Universidad Palmas de Gran Canaria. https:/tig.age-geografia.es/wp-content/ uploads/2021/09/GranCanaria2008r.pdf
Rivera Forero, L. J. (2014). Fuente de energía, renovable y no renovable. Revista Escuela de Administración de Negocios, (77), 216-218. https://www.redalyc.org/ pdf/206/20633274015.pdf

Rodríguez Murcia, H. (2008). Desarrollo de la energía solar en Colombia y sus perspectivas. Revista de Ingeniería, (28), 83-89. http:// www.scielo.org.co/pdf/ring/n28/n28a12.pdf

Sabogal Aguilar, J. y Hurtado Aguirre, E. (2009). La historia se repite: una visión del desarrollo y del desarrollo sostenible. Revista de la Facultad de Ciencias Económicas, 17(1), 195-216. http://www.scielo.org. co/pdf/rfce/v17n1/v17n1a13.pdf

Vilches, A. y Gil Pérez, D. (2012). La educación para la sostenibilidad en la universidad: el reto de la formación del profesorado. Profesorado. Revista de Curriculum y Formación de Profesorado, 16(2), 25-43. https://www. redalyc.org/pdf/567/56724395003.pdf 